\title{
Effect of Zinc Doped in Cobalt Ferrite Nano Particles Prepared by Chemical Co-Precipitation Method
}

\author{
B.SURYANARAYANA ${ }^{1 *}$ VEMURI RAGHAVENDRA $^{1}$ \\ K.CHANDRA MOULI ${ }^{1}$ and P.RAGHUPATHIREDDY ${ }^{2}$
}

${ }^{1}$ Solid State Physics \& Materials Research Labs, Dept.of Engineering Physics,
Andhra University, Visakhapatnam-530 003, India
${ }^{2}$ Anurag Group of Institutions, Hyderabad, India
suryanarayana.badireddi@gmail.com

Received 23 May 2016 / Accepted 4 June 2016

\begin{abstract}
Zinc doped in cobalt ferrite with chemical formula $\mathrm{Co}_{1-\mathrm{x}} \mathrm{Zn}_{\mathrm{x}} \mathrm{Fe}_{2} \mathrm{O}_{4}$ with $\mathrm{x}=(0.15,0.25$, $0.5,0.55,0.7)$ was synthesized by chemical co-precipitation method.The values of coercivity decreased from $104 \mathrm{Oe}$ to $0.505 \mathrm{Oe}$ as $\mathrm{Zn}$ content increased from 0.15 to 0.7 . It is observed that the magnetization reminisce ratio has decreased from 0.0842 to 0.00027 at room temperature as the $\mathrm{Zn}$ content increased to 0.7 , suggests that the nano particles exhibit an effective anisotropy.
\end{abstract}

Keywords: Magnetic studies, Coercivity, Cobalt ferrite, Chemical Co-Precipitation method, Nano particles

\section{Introduction}

These properties make it a promising material for high density magnetic recording, permanent magnets, magnetic fluids, photo-magnetic material ${ }^{1}$ and gas sensing ${ }^{2}$. The size reduction of a magnetic material leads to novel properties like superparamagnetism as compared to bulk material due to small volume ${ }^{3}$. Therefore an understanding of the structural and magnetic properties of spinels is of great importance from both a fundamental and an applied filed. $\mathrm{CoFe}_{2} \mathrm{O}_{4}$ particles with spinel structure show a variety of novel properties that vary with the nature of the cations, their charge, and site distribution amongst tetrahedral and octahedral sites. For this reason, different experimental methods have been used in the production of ferrite nanoparticles such as sol-gel techniques ${ }^{4}$, co-precipitation method $^{5-6}$ microwave sintering method ${ }^{7}$, spray-spin-heating-coating method ${ }^{8}$ and sonochemical method $^{9}$. In this paper mainly discuss the magnetic studies and curie temperature of zinc doped in cobalt ferrite by Co-precipitation method. 


\section{Experimental}

In our studies $\mathrm{Co}_{1-\mathrm{x}} \mathrm{Zn}_{\mathrm{x}} \mathrm{Fe}_{2} \mathrm{O}_{4}$ (where $\mathrm{x}=0.15,0.25,0.5,0.55,0.7$ ) ferrite compositions were prepared by chemical co-precipitation method $^{5}$ by using high purity chemicals Ferric chloride $\left(\mathrm{FeCl}_{3}\right)$, Cobalt chloride $\left(\mathrm{CoCl}_{2}\right)$, Zinc chloride, Sodium Hydroxide $(\mathrm{NaOH})$ as starting materials. Fine nanoparticles of ferrites were prepared by chemical co-precipitation method ${ }^{6}$. These chlorides were weighed in the required stoichiometric proportions and dissolved in distilled water and $\mathrm{NaOH}$ solution was added to this aqueous solution under continuous stirring at constant speed. The reaction temperature was maintained constant of $45{ }^{\circ} \mathrm{C}$ while mixing the alkaline aqueous solution. Therefore, precipitation occurred immediately and the color of suspension changed from brown to dark brown. The mixture was stirred for $2 \mathrm{~h}$ at constant temperature of $45^{\circ} \mathrm{C}$. The precipitation was washed with distilled water to remove sodium and chlorine ions. The washed samples were dried in air for $20 \mathrm{~h}$. The dried powders were mixed with $5 \mathrm{wt} \%$ polyvinyl alcohol and ground well, which were compacted using uni axial hydraulic pressing machine (with a pressure of 8-9 tonnes) and green bodies (1.7 $\mathrm{mm}$ in thickness and $6 \mathrm{~mm}$ in diameter) were sintered at $1000{ }^{\circ} \mathrm{C}$ for $1 \mathrm{~h}$ with steady rate of heating of $50{ }^{\circ} \mathrm{C}$ per hour in natural furnace chamber atmosphere to obtain sintered disks. These dried powders were analyzed for phase analysis by powder x-ray diffraction.

\section{Results and Discussion}

\section{Magnetic studies}

The room temperature magnetization measurements have been carried out using a Vibrating Sample Magnetometer (ADE Technologies, USA; Model: 1660 MRS). We measured magnetization as a function of applied magnetic field for $\mathrm{Co}_{1-\mathrm{x}} \mathrm{Zn}_{\mathrm{x}} \mathrm{Fe}_{2} \mathrm{O}_{4}$ products for all the compositions using magnetic field ranging from 0 to $20 \mathrm{kO} e$ at room temperature, Figure 1 shows the room temperature hysteresis loop of the prepared powder samples. The magnetization curves show neither hysteresis nor coercivity. This is characteristic of superparamagnetic behaviour. The $\mathrm{M}-\mathrm{H}$ loops observed in the present study show that the cation distribution has changed from the normal to the mixed spinal type. Hence some percentage of $\mathrm{Fe}^{3+}$ is pushed to the tetrahedral sites, which switches on the A-B superexchange interaction between $\mathrm{Fe}^{3+}$ ions on both sites and gives rise to ferromagnetic ordering. The unsaturated magnetization behavior observed in nanocrystalline zinc ferrite reveals the presence of a superparamagnetic and single domain nature of the particles ${ }^{10}$.

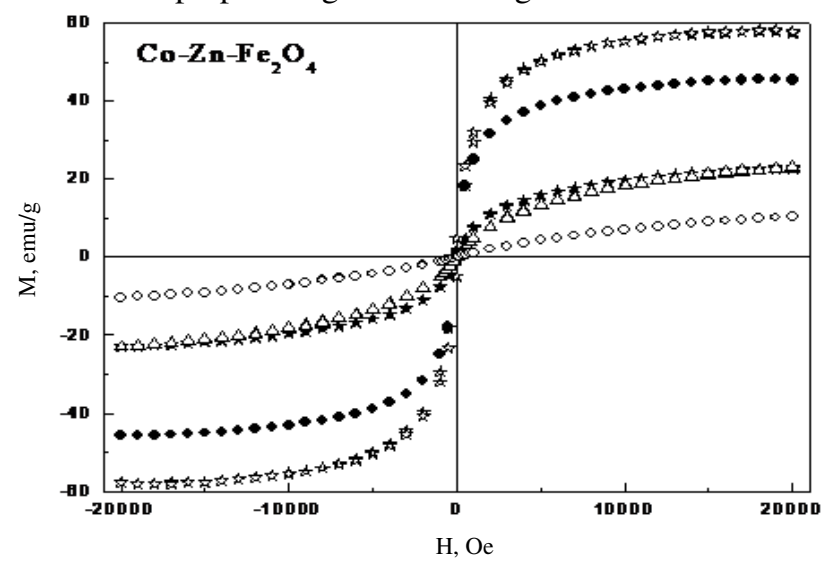

Figure 1. Hysteresis behaviour 
Table 1. Magnetic data for the Co- $\mathrm{Zn}-\mathrm{Fe}_{2} \mathrm{O}_{4}$ nanoferrites

\begin{tabular}{cccccc}
\hline Composition & $\mathrm{M}_{\mathrm{s}}$ emu/g & $\mathrm{M}_{\mathrm{r}}$ emu/g & $\mathrm{M}_{\mathrm{r}} / \mathrm{M}_{\mathrm{s}}$ & $\mathrm{H}_{\mathrm{c}} \mathrm{Oe}$ & $\mathrm{Tc}$ \\
\hline $\mathrm{Co}_{0.85} \mathrm{Zn}_{0.15} \mathrm{Fe}_{2} \mathrm{O}_{4}(\mathrm{~S}-\mathrm{I})$ & 57.06 & 4.810 & 0.08429 & 104.50 & 290 \\
$\mathrm{Co}_{0.75} \mathrm{Zn}_{0.25} \mathrm{Fe}_{2} \mathrm{O}_{4}(\mathrm{~S}-\mathrm{II})$ & 46.75 & 1.450 & 0.03101 & 38.28 & 225.5 \\
$\mathrm{Co}_{0.5} \mathrm{Zn}_{0.5} \mathrm{Fe}_{2} \mathrm{O}_{4}(\mathrm{~S}-\mathrm{III})$ & 22.03 & 0.028 & 0.00127 & 2.871 & 149 \\
$\mathrm{Co}_{0.45} \mathrm{Zn}_{0.55} \mathrm{Fe}_{2} \mathrm{O}_{4}(\mathrm{~S}-\mathrm{IV})$ & 22.00 & 0.006 & 0.00027 & 0.831 & 78 \\
$\mathrm{Co}_{0.3} \mathrm{Zn}_{0.7} \mathrm{Fe}_{2} \mathrm{O}_{4}(\mathrm{~S}-\mathrm{V})$ & 11.40 & 0.005 & 0.00043 & 0.505 & 51 \\
\hline
\end{tabular}

The graph between saturation magnetization $\left(\mathrm{M}_{\mathrm{s}}\right)$ as the $\mathrm{Zn}$ content is shown in Figure 2. From the graph, $\mathrm{M}_{\mathrm{s}}$ decrease linearly as the $\mathrm{Zn}$ concentration increases. The saturation magnetization was found to decrease from 57.06 to $11.40 \mathrm{emu} / \mathrm{g}$ when the concentration of Zinc was increased from 0.15 to 0.7 . Ms of Co-Zn ferrites decrease with $\mathrm{Zn}$ content due to the strong covalency effects arising from small cationic site dimensions. The saturation magnetization decreases with $\mathrm{Zn}$ increased, since the diamagnetic $\mathrm{Zn}^{2+}$ ions replace the magnetic $\mathrm{Co}^{2+}$ ions at tetrahedral sites due to the prominent inter-sub lattice A-B superexchange interactions. At room temperature, the value of Ms obtained for our Nanoparticles of $\mathrm{Co}_{0.85} \mathrm{Zn}_{0.15} \mathrm{Fe}_{2} \mathrm{O}_{4}$ is $57.06 \mathrm{emu} / \mathrm{g}$ and which is lower than $66 \mathrm{emu} / \mathrm{g}$ for the $\mathrm{CoFe}_{2} \mathrm{O}_{4}$ bulk ${ }^{11}$. But this value is marginally matching with $\mathrm{CoFe}_{2} \mathrm{O}_{4} 58.3 \mathrm{emu} / \mathrm{g} \mathrm{Kim} \mathrm{et} \mathrm{al.}{ }^{12}$.

The remanent magnetization $\mathrm{Mr}$ with respect to the $\mathrm{Zn}$ content change is shown in Figure. From the graph it is observed that the value of remanent magnetization decreases as a function of the $\mathrm{Zn}$ content change. The decrement of the values of remanent magnetization $\mathrm{M}_{\mathrm{r}}$ is due to the influence of the cationic stochiometry and their occupancy, in the specific sites. The value of squareness ratio $\left(\mathrm{M}_{\mathrm{r}} / \mathrm{M}_{\mathrm{s}}\right)$ is decreases as the $\mathrm{Zn}$ content increases as shown in Figure 2. This indicates that the system consists of randomly oriented equiaxial particles with cubic magneto crystalline anisotropy ${ }^{13}$. It is well known that the fine particles are easier to be thermally activated to overcome the magnetic anisotropy ${ }^{14}$. When size of the nanoparticles is smaller than the critical size, the blocking temperature, the hysteresis behavior vanishes and the magnetization direction of the particles simply follows the direction of the applied magnetic field. The hysteresis loops demonstrate a typical super paramagnetic behavior with very low coercivity and remanence. The existence of an effectively uniaxial anisotropy in magnetic nanoparticles has been attributed to surface effects ${ }^{15}$ as evidenced by simulations of nanoparticles. Surface effects also tend to lead large anisotropies. The interaction between the core and surface spins leads to a variety of effects including large anisotropy and exchange bias effects.

The values of coercive fields are found to decrease linearly with $\mathrm{Zn}$ content increases as shown in Figure 3. But it is interesting to note that the coercivity (103.50 Oe) obtained is lower when compared to the coercivity of cobalt ferrites $\left(193\right.$ Oe) reported earlier by Kim et al. ${ }^{16}$ The reduction in the magnetic property may be due to the partial substitution of nonmagnetic $\mathrm{Zn}$, due to size effect or due to the presence of super para mgnetic particles. The coercivity is found to be maximum when the partial substitution of zinc with cobalt ferrite is minimum. The substitution of a non magnetic ion such as $\mathrm{Zn}$, which has preferential site occupancy, results in the reduction of the exchange interaction between A and B sites. In these types of ferrite materials, earlier reports ${ }^{17}$ indicate the behavior of super paramagnetic behavior but in our results showed ${ }^{18}$ a clear evidence of ferromagnetic behavior ${ }^{19}$ which is very suitable for spintronics applications in the case of hard disk storage devices ${ }^{20}$. This coercivity decrease at large particle sizes could be attributed to either of two reasons. First, it may be due to the expected crossover from single domain to multi-domain behavior with increasing size. Second such an effect can arise from a combination of surface anisotropy and thermal energies. We understand the decrease of coercivity with increasing $\mathrm{Zn}$ content 
as being due to the enhanced role of the surface and its strong anisotropy as opposed to the bulk anisotropy. This rise is followed by a decline at small enough sizes when the product of anisotropy energy and volume becomes comparable to the thermal energy, however the dominant role will be of the surface effects for smaller particles.

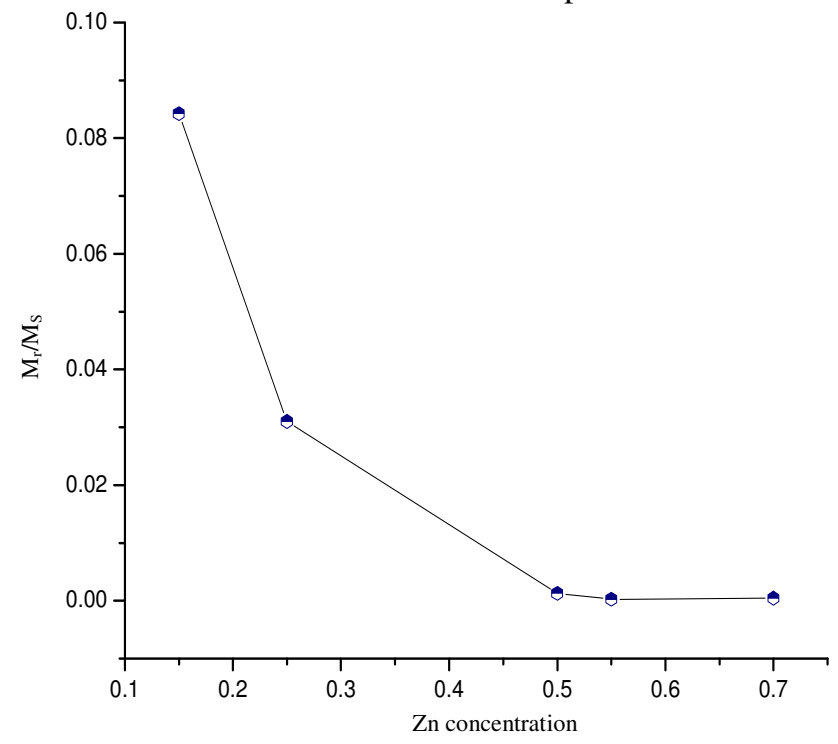

Figure 2. Variation of $\mathrm{M}_{\mathrm{r}} / \mathrm{M}_{\mathrm{s}}$ ration with $\mathrm{Zn}$ content change

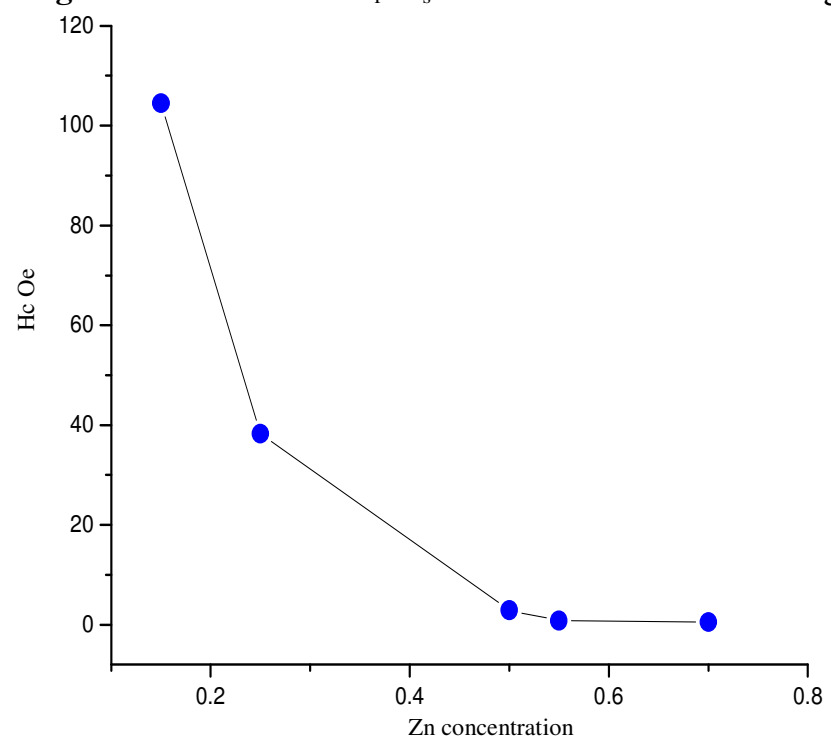

Figure 3. Variation of coercivity of $\mathrm{Zn}$ content change

Curie temperature studies

The Curie temperature measurements have been carried out using a pulse field magnetometer (PFM) a house made instrument at Ferrite Laboratory, RRCAT, India. From the PFM instrument, we measured specific magnetization as function of temperature as 
shown in Figure 4. The measurements were performed in the range of temperature from $5{ }^{\circ} \mathrm{C}$ to $400{ }^{\circ} \mathrm{C}$. The specific magnetization decreases with the increase of the partial substitution of zinc with cobalt. This is typical behavior for superparamagnetic materials as a result of the decrease in the thermal energy ${ }^{21}$. The variation of specific magnetization as function of temperature of $\mathrm{Co}_{1-\mathrm{x}}$ $\mathrm{Zn}_{\mathrm{x}} \mathrm{Fe}_{2} \mathrm{O}_{4}(\mathrm{x}=0.15,0.25,0.55)$ nano particles is shown in Figure 4. From the graphs, it is observed that the Curie temperature decreases with the increase of the $\mathrm{Zn}$ content. This decrease of the Curie temperature, which is due to the increase of the nonmagnetic $\mathrm{Zn}$ substitution with the cobalt, can bring down the Curie temperature close to the room temperature ${ }^{22}$.
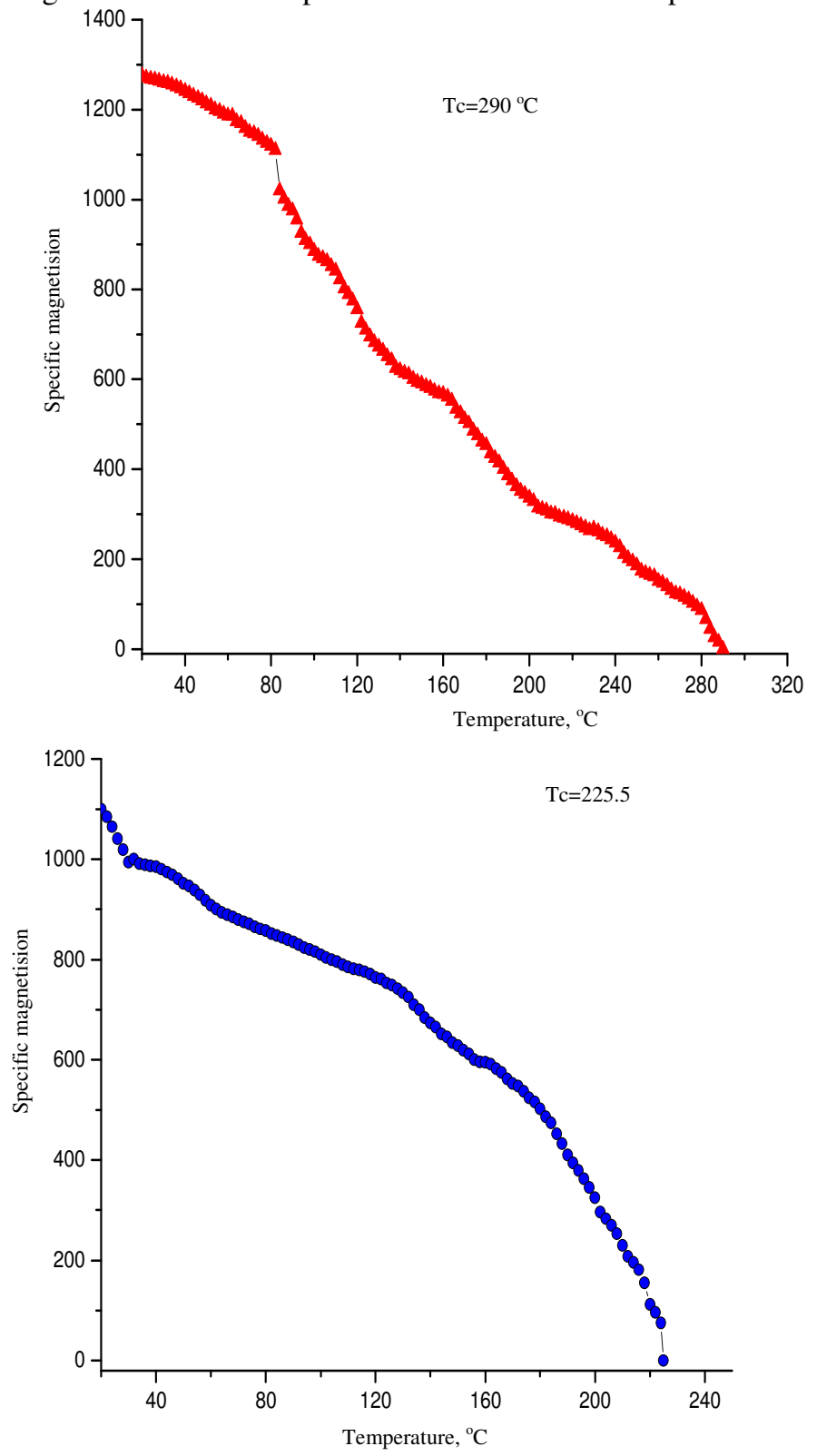


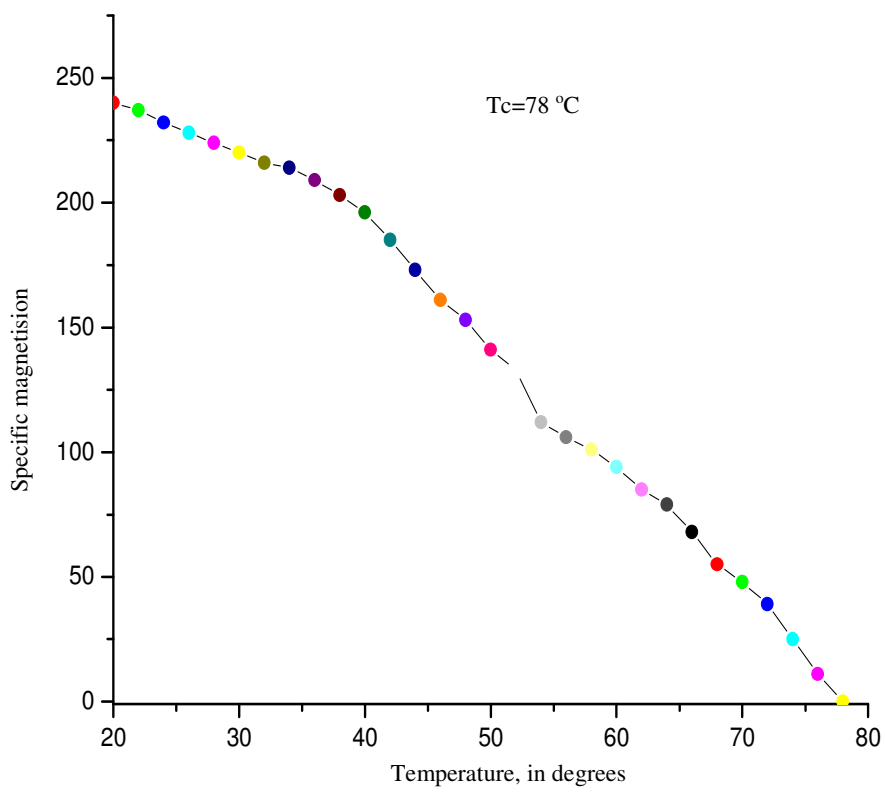

Figure 4. The variation of specific magnetization as function of temperature $\mathrm{Co}_{1-\mathrm{x}} \mathrm{Zn}_{\mathrm{x}} \mathrm{Fe}_{2} \mathrm{O}_{4}$ $(\mathrm{x}=0.15,0.25,0.55)$ nano particles

\section{Conclusion}

The saturation magnetization, which decreased from 57.06 to $11.40 \mathrm{emu} / \mathrm{g}$ for the increase of $\mathrm{Zn}$ content from 0.15 to 0.7 moles at room temperature appears to be due to pronounced growth of magnetic anisotropy. It is observed that the magnetization reminisce ratio has decreased from 0.0826 to 0.00027 at room temperature as the $\mathrm{Zn}$ content increased to 0.7 , suggests that the nano particles exhibit an effective anisotropy.

\section{References}

1. Zhao D, Wu X and Guan H, J Supercritical Fluids, 2007, 42(2), 226-233; DOI:10.1016/j.supflu.2007.03.004

2. Giri A K, Kirkpatric E M, Moongkhmkla P, Majetich S A and Harris V G, Appl Phys Lett., 2002, 80(13), 2341; DOI:10.1063/1.1464661

3. Bueno A R, Gregori M L and Nóbrega M C S, Mater Chem Phys., 2007, 105(2-3), 229-233; DOI:10.1016/j.matchemphys.2007.04.047

4. Manju Kurian, Smitha Thankachan, Divya S Nair, Aswathy E K, Aswathy Babu, Arathy Thomas and Binu Krishna K T, J Advd Ceramc.,, 2015, 4(3), 199-205; http://dx.doi.org/10.1007/s40145-015-0149-x

5. Suryanarayan B, Vemuri Raghavendra and Chandra Mouli K, Chem Sci Trans., 2016, 5(1), 137-144; DOI:10.7598/cst2016.1166

6. Suryanarayana B, Vemuri Raghavendra and Chandra Mouli K, Int Lett Chem., Phys Astronomy, 2015, 60, 20-24; DOI:10.18052/www.scipress.com/ILCPA.60.20

7. Feng H, Chen B and Zhang D, Zhang J and Tan L, J Magn Magn Mater., 2014, 356, 68-72; DOI:10.1016/j.jmmm.2013.12.033

8. $\quad$ Kurian M and Nair D S, J Environ Chem Engg., 2014, 2(1), 63-69;

DOI:10.1016/j.jece.2013.11.026 
9. Sorescu M, Diamandescu L and Peelamedu R, J Magn Magn Mater., 2004, 279(2-3), 195-201; DOI:10.1016/j.jmmm.2004.01.079

10. Bean C P and Livingston J D, J Appl Phys., 1959, 30, 1208.

11. Bate G, in: Wohlfarth E P, (Ed.), Ferromagnetic Materials, vol. 2, North-Holland, Amsterdam, 1980, p. 431.

12. Y. II Kim, D. Kim, C.S. Lee, Physica B, 2003, 337, 42.

13. Berkowitz A E and Kneller E, Magnetism and Metallurgy (Academic, New York, 1969, Vol. 1, Chap.8.

14. Xuebo Cao and Li Gu, Nanotechnology, 2005, 16, 180-185; DOI:10.1088/0957$4484 / 16 / 2 / 002$

15. Kodama R H, J Mag Mag Mater., 1999, 200(1-3), 359-372; DOI:10.1016/S03048853(99)00347-9

16. Sato T, Iijima T, Seki M and Inagaki N, J Magn Magn Mater., 1987, 65(2-3), 252256; DOI:10.1016/0304-8853(87)90044-8

17. Chen Q and Zhang Z L, Appl Phys Lett., 1998, 73, 3156-3158; DOI:10.1063/1.122704

18. Bean C P and Livingston J D, J Appl Phys., 1959, 30, 120; DOI:10.1063/1.2185850

19. Wang Z L, Liu Y and Zhang Z, (Ed.), (2003) Handbook of Nanophase and Nanostructured Material (New York: Kluwer-Academic).

20. Bate G, in: Wohlfarth E P, (Ed.), Ferromagnetic Materials, vol. 2, North-Holland, Amsterdam, 1980, p. 431.

21. Dong H C and Chen Y Y, Mater Res Bull., 2002, 37(4), 801-810; DOI:10.1016/S0025-5408(01)00590-6

22. Arulmurugan R, Vaidyanathan G, Sendhilnathan S and Jeyadevan B, Physica B, 2005, 363(1-4), 225--231; DOI:10.1016/j.physb.2005.03.025 